\title{
On the Thermodynamical Theory of Thermal Conduction of Dielectrics Under Electric Fields.
}

\author{
R. FIESCHI \\ Istituto di Scienze Fisiche dell'Università - Milano \\ Istituto Nazionale di Fisica Nucleare - Sezione di Milano \\ (ricevuto il 4 Luglio 1957)
}

In a recent paper ( ${ }^{1}$ ), by means of the methods of the thermodynamical theory of irreversible processes $\left({ }^{2}\right)$, the following expression has been derived for the thermal conductivity of dielectries in the presence of a longitudinal electric field:

$$
k=k_{0}+\frac{\left(\sigma-\sigma_{0}\right) \theta}{\left(\theta_{s}-\theta\right)^{2}} \nabla^{2}
$$

The conclusions drawn by the author, that $k$ does not depend on the sign of the field and that the dependence of $k$ on $V$ is quadratic, however do not hold since $\Delta \sigma \equiv \sigma-\sigma_{0}$ depends on the re Itive orientation of thermal and electrical potential gradients. Indeed from the phenomenological equations it follows:

$$
\Delta \sigma(V, \operatorname{grad} \theta)=-\Delta \sigma(-V, \operatorname{grad} \theta)=L_{12} \frac{\theta_{s}-\theta}{\theta V},
$$

hence, from (1)

$$
\Delta k(V, \operatorname{grad} \theta)=-\Delta k(-V, \operatorname{grad} \theta)=L_{12} \frac{V}{\theta_{3}-\theta},
$$

(see Fig. 1 and 2).

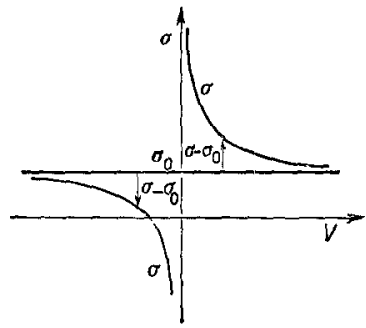

Fig. 1.

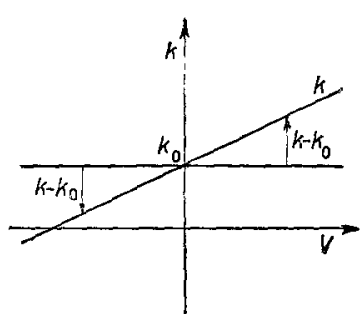

rijğ 2.

The correspondence with Dr. MascaRenhas is acknowledged.

(1) S. Masmaresuas Nuovo Cimento, 5, 1118 (1957).

(2) See, for instance, S. R. DE GRoc $\mathrm{T}$ : Thermociynamics of Irreversiule Processes (Amsterdam, 1951). 